\title{
Effects of Different Cytoprotectants Combination on Sperm Survival, Fertility and Embryo Development in Amur Sturgeon (Acipenser schrenckii)
}

\author{
Xiujuan Zhang, Jinge Ma, Linmiao Li, Haiying Jiang, Jinping Chen* \\ Guangdong Key Laboratory of Animal Conservation and Resource Utilization, Guangdong Public Laboratory of Wild Animal Conservation \\ and Utilization, Guangdong Institute of Applied Biological Resources, Guangzhou, China
}

Email address:

chenjp@giabr.gd.cn (Jinping Chen)

${ }^{*}$ Corresponding author

\section{To cite this article:}

Xiujuan Zhang, Jinge Ma, Linmiao Li, Haiying Jiang, Jinping Chen. Effects of Different Cytoprotectants Combination on Sperm Survival, Fertility and Embryo Development in Amur Sturgeon (Acipenser schrenckii). Animal and Veterinary Sciences. Vol. 6, No. 1, 2018 , pp. 51-57. doi: 10.11648/j.avs.20180604.11

Received: August 4, 2018; Accepted: September 4, 2018; Published: October 13, 2018

\begin{abstract}
Development of artificial propagation technology is becoming increasingly important in sturgeon aquaculture whether species recovery efforts or commercial production. The cryopreservation technique of high-quality semen collected during the spawning season could extremely improve reproductive efficiency for year-round availability, especially for off-season use. However, the cryopreservation technique of sperm in Amur sturgeon (Acipenser schrenckii) has not been effectively developed. In the present study, we firstly tested the cryopreservations combination effects of three cryoprotectants, including methanol, dimethyl sulfoxide (DMSO), and propylene glycol (PG) and fresh yolk (Y) addition on sperm motility, fertilization and embryo development in Amur sturgeon. The results indicated that sperm motility was still more than $60 \%$ in the MT group but that of the control group was sharply decreased to $4.5 \%$ after $72 \mathrm{~h}$ of in vitro storage $\left(4^{\circ} \mathrm{C}\right)$. The post-thawed sperm motility analysis showed that there was no significant difference between the MT+DMSO group and MT group, but the fertility rate in the MT group was significantly higher with a value of $42.30 \pm 2.57(\%)$ than any other experimental groups, including the MT+DMSO group $(\mathrm{P}<0.05)$. Meanwhile, we also found that there was no significantly positive effect on post-thawed sperm motility with Y addition. Interestingly, although the results showed that the MT+DMSO group and MT group had similar effects on the post-thawed sperm motility, the MT+DMSO group had higher hatching rate compared to any other tested groups, including the MT group. Meanwhile, we also found that PG as cryoprotectant was unsuitable for sperm cryopreservation of Amur sturgeon. In conclusion, our results provides invaluable basis in further studies for the optimization technology of artificial propagation in Amur sturgeon.
\end{abstract}

Keywords: Acipenser schrenckii, Cryoprotectant, Sperm Cryopreservation, Embryo Development

\section{Introduction}

Sturgeons are polyploid chondrostean fish that originated during the Devonian period and belong to the order Acipenseriformes, which contains 27 species divided into two families: Acipenseridae (sturgeon, 25 species) and Polyodontidae (paddlefish, two species). Sturgeon caviar is praised as "black gold" and represents one of the most highly prized foods of animal origin [1]. Therefore, wild resource of most sturgeon species has been greatly impacted by anthropogenic factors, for example, habitat destruction and overexploitation. According to the International Union for Conservation of Nature Resource (IUCN) Red List, $85 \%$ of all sturgeon species are identified as one of the most endangered groups of animals and on the verge of extinction in the January 2018 edition (www.iucnredlist.org/). Interestingly, the conflict between the severe decline in natural populations and the huge profits from commercial sturgeon farming is the main driving force of the development of sturgeon aquaculture. Currently, more than 17 sturgeon species have been farmed and China 
accounts for $15 \%$ of total sturgeon production [2]. Although the rapid development of sturgeon aquaculture has greatly eliminated the pressure on wild stocks, artificial propagation technology in sturgeon is still increasingly important for species recovery efforts as well as commercial production.

Amur sturgeon (Acipenser schrenckii) is a species of fish belonging to the Acipenseridae family and is found in the Amur River, Songhua River, and Heilong River [3]. Amur sturgeons are commonly used as hybridization parents of sturgeon aquaculture. However, one difficulty in hybridization reproduction is the synchronization of gamete availability from both sexes of the two different sturgeon species. Although off-season spawning can be induced by manipulating various measures, such as the temperature cycles, photoperiod, and sex-hormone stimulation, these techniques are costly and low efficiency. Moreover, the survival rate and fertility rate of sperm are often dramatically decreased in aquaculture practice. Therefore, if high-quality semen collected during the spawning season could be cryopreserved for year-round availability, especially for off-season use, this will extremely improve reproductive efficiency. However, the cryopreservation technique of sperm in Amur sturgeon has not been effectively developed.

Vitrification cryopreservation has been recommended to increase the probability of success, and the effective preservation protocols require higher concentrations of cryoprotectants [4, 5]. To date, the methods of sperm cryopreservation have been developed in many sturgeon species, including Acipenser baeri [6], A. ruthenus [7], Huso huso [8] and A. persicus [9]. However, these studies also showed that the cryopreservation effects of different frozen extenders on sperm of sturgeon species were varied considerably. Although the frozen extenders based on methanol as cryoprotectant addition achieved the better survival rates of post-thaw sperm in many sturgeon species, the index of fertilization rate was remarkably unstable (Table 1). The latest report indicated that cryoprotectants combination were suitable for cryopreservation of sturgeon embryos [10], which might suggest that cryoprotectants combination of sperm cryopreservation in sturgeon may be more suitable method. Therefore, the present study was conducted to test the effects of different cryoprotectants combination on sperm survival, fertility and subsequent embryo development in Amur sturgeon.

Table 1. Comparison of some semen characteristics in sturgeon species following freezing-thawing process.

\begin{tabular}{|c|c|c|c|c|c|}
\hline Species & Extender / Cryoprotectant & Freezing procedure & $\begin{array}{l}\text { Sperm } \\
\text { motility (\%) }\end{array}$ & $\begin{array}{l}\text { Fertility } \\
\text { rate }(\%)\end{array}$ & references \\
\hline Siberian sturgeon (A.baeri) & Tris-sucrose- $\mathrm{KCl}^{\mathrm{a}} / 10 \% \mathrm{MT}$ & programmable freezer & $\mathrm{N}^{\mathrm{b}}$ & Over $80 \%$ & Glogowski et al., 2002 \\
\hline Paddlefish (Polyodon spathula) & $\begin{array}{l}\text { modified Tris-sucrose- } \mathrm{KCl}^{\mathrm{c}} \\
/ 8-10 \% \mathrm{MT} \text { or DMSO }\end{array}$ & programmable freezer & $70-86 \%$ & $64-75 \%$ & Linhart et al.,2006 \\
\hline Beluga sturgeon (Huso huso) & Tris-sucrose- $\mathrm{KCl} / 10 \% \mathrm{MT}$ & programmable freezer & $52-70 \%$ & $55-67 \%$ & Aramli et al., 2015 \\
\hline Persian sturgeon $(A$. persicus $)$ & Tris-sucrose- $\mathrm{KCl} / 10 \% \mathrm{MT}$ & programmable freezer & $60 \pm 4.1 \%$ & $72 \pm 8.36 \%$ & Aramli et al., 2016 \\
\hline $\begin{array}{l}\text { Shortnose sturgeon } \\
\text { (A.brevirostrum) }\end{array}$ & $\begin{array}{l}\text { modified Tris-sucrose- } \mathrm{KCl}+ \\
\text { osmolality: } 82 \mathrm{mosM} / \mathrm{kg} / 5 \% \\
\text { DMSO }\end{array}$ & $\begin{array}{l}3 \mathrm{~cm} \text {-high above liquid } \\
\text { nitrogen level, } 3 \mathrm{~min} ;\end{array}$ & $26 \pm 13 \%$ & 0 & Horvath et al., 2005 \\
\hline Sterlet (A. ruthenus) & Tris-sucrose- $\mathrm{KCl} / 10 \% \mathrm{MT}$ & $\begin{array}{l}3 \mathrm{~cm} \text {-high above liquid } \\
\text { nitrogen level , 20min; }\end{array}$ & $5-67 \%$ & $13-76 \%$ & Dzyuba et al., 2012 \\
\hline Amur sturgeon (A. schrenckii) & $\begin{array}{l}\text { modified Tris-sucrose- } \mathrm{KCl}+ \\
\mathrm{BSA}+\text { Glutathione } / 10 \% \mathrm{MT}\end{array}$ & $\begin{array}{l}\text { 10cm-high above liquid } \\
\text { nitrogen level , 5-10min; }\end{array}$ & $37.3 \pm 6.4 \%$ & $42.3 \pm 2.6 \%$ & The present study \\
\hline
\end{tabular}

${ }^{\mathrm{a}}$ indicates Tris-sucrose- $\mathrm{KCl}(30 \mathrm{mM}$ Tris, $23.4 \mathrm{mM}$ sucrose, $0.25 \mathrm{mM} \mathrm{KCl}, \mathrm{pH} 8.0)$.

${ }^{\mathrm{b}}$ represents no statistics in the report.

${ }^{\mathrm{c}}$ indicates modified Tris-sucrose- $\mathrm{KCl}$ (20 mM Tris, $30 \mathrm{mM}$ sucrose, $0.5 \mathrm{mM} \mathrm{KCl}, \mathrm{pH} 8.0$ ).

MT indicates abbreviation of methanol.

\section{Materials and Methods}

\subsection{Ethics}

All experimental animal procedures followed the principles of the Guide for Care and Use of Laboratory Animals and were approved by the Animal Experimental Ethical Committee of Guangdong Institute of Applied Biological Resources.

\subsection{Fish and Gamete Collection}

All experiments were performed in the Engineering and Technology Center of Sturgeon Breeding and Cultivation of the Chinese Academy of Fishery Science (Beijing, China) during April and May 2016. Three adult females (IV stage; total length, 142-162 cm) and three adult males (good records; total length, 139-158 cm) were chosen and transferred to the breeding workshop for the synchronous breeding procedure. In brief, after water temperature stimulation of $14^{\circ} \mathrm{C}-16^{\circ} \mathrm{C}$ for 10 day in tanks with an oxygen concentration $>5 \mathrm{mg} / \mathrm{L}$ and a $\mathrm{pH}$ of 7.6-7.9, the parent fish received an injection of the luteinizing hormone-releasing hormone analogue (LHRH) into their pectoral fin muscle. Spermiation in males was stimulated by the LHRH dose of $2.5 \mu \mathrm{g} / \mathrm{kg}$ that occurred $36 \mathrm{~h}$ before semen collection, and semen were collected with urogenital papilla by aspiration through a plastic catheter (1-2 $\mathrm{cm}$ diameter) connected to a $500-\mathrm{mL}$ sterile plastic bottle. Sperm motility $>90 \%$ was considered to be a qualified sample for further analysis. Ovulation in females was stimulated by the LHRH at $5 \mu \mathrm{g} / \mathrm{kg}$ using two injection during a 12-hour interval; at the first injection, $20 \%$ of the dose was provided, and the remaining $80 \%$ provided in the second injection. The 
mature degree of the egg was evaluated $24 \mathrm{~h}$ after the last injection.

\subsection{Cryopreservation Protocol}

The semen volume was measured, and sperm density was estimated using a cell haemocytometer at $200 \times$ magnification on a contrast microscope (Olympus, Tokyo, Japan). Three frequently used cryoprotectants-methanol (MT), DMSO, and propylene glycol (PG)-were used in the following experiments. The base medium (BM) of frozen extender which were mainly composed of $23.4 \mathrm{mM}$ sucrose, $0.25 \mathrm{mM}$ $\mathrm{KCl}, 30 \mathrm{mM}$ Tris- $\mathrm{HCl}, 10 \mathrm{~g} / \mathrm{L}$ BSA, $5 \mathrm{mM}$ glutathione and had a $\mathrm{pH}$ value of 8.0, was modified according to previous methods [2].Meanwhile, fresh yolk (Y) was also considered addition in the protocols of sperm cryopreservation for $A$. schrenckii. Therefore, we designed six groups: 1) BM $+10 \%$ MT (MT group); 2) $\mathrm{BM}+10 \% \mathrm{MT}+10 \% \mathrm{DMSO}$ (MT + DMSO group); 3) $\mathrm{BM}+10 \% \mathrm{MT}+10 \% \mathrm{DMSO}+10 \% \mathrm{Y}$ (MT + DMSO + Y group); 4) BM + 10\% MT + 10\% PG (MT $+\mathrm{PG}$ group); 5) $\mathrm{BM}+10 \% \mathrm{MT}+10 \% \mathrm{PG}+10 \% \mathrm{Y}(\mathrm{MT}+$ $\mathrm{PG}+\mathrm{Y}$ group); and 6) control group (fresh semen).

All the cryopreservation media were precooled overnight at $4^{\circ} \mathrm{C}$ of a refrigerator. Qualified semen was respectively diluted $1: 1$ with each of the five above-mentioned medium groups. To test toxicity of different cryoprotectants combination, the parts of each of the diluted semen of five different groups and undiluted control group were continued to be placed at $4{ }^{\circ} \mathrm{C}$ of a refrigerator for further observation. In order to examine the frozen effects, majority of diluted semen were filled with 0.5-mL CBSTM cryobiostraws using micro-classic suction device (Cryo Bio System, Legler, France). Then cryobiostraws were immediately collected into CBSTM plastic cups and performed the freezing procedures, which included the following steps: first, the samples were balanced for 5-10 min on an ice-water mixture $\left(0^{\circ} \mathrm{C}\right)$. Then, they were placed in $10 \mathrm{~cm}$ above liquid nitrogen for 5-10 min. Finally, the cryobiostraws were immediately immersed into liquid nitrogen $\left(-196^{\circ} \mathrm{C}\right)$ for long-term storage.

\subsection{Evaluation of Sperm Motility}

To examine the frozen effects, each cryobiostraw from all the tested groups was immediately thawed for 3-5 sec in a $37^{\circ} \mathrm{C}$ water bath and the motility parameter was re-evaluated. Tris- $\mathrm{HCl}$ buffer $(10 \mathrm{mM}, \mathrm{PH}=8.0)$ was used as the activating medium (AM) according to the previous method of beluga sturgeon [8]. Sperm motility was recorded by dark-filed microscopy (Olympus, Tokyo, Japan). The motility percentage of each experimental group was determined on a $0-10$ point scale, where 0 represented $0 \%$ motility and 10 denoted $100 \%$ motility. One person conducted all sperm motility observations to reduce the degree of variation.

\subsection{Fertilization Ability and Embryo Development Assay}

Equal volumes of eggs from the three females were pooled. Each group was allocated $30 \mathrm{~g}$ of eggs and three replicates (approx. 500 eggs / 10 g) were also designed for each group. Freshly collected and allocated eggs were immediately inseminated with post-thawed sperms from different groups. The number of fertility embryos was counted after $44 \mathrm{~h}$ incubation. Therefore, the fertility rate was denoted as the proportion of fertile embryos from the number of initial eggs. The number of hatched fish in each group was counted on the first day of fry emergence. The hatching rate was counted as the proportion of the number of hatched fish from the number of initial eggs.

\subsection{Statistical Analyses}

The mean and standard deviation (mean \pm SD) were calculated from the technological and biological replication. The statistical significance among the different groups were measured using the method of one-way ANOVA with Fisher's LSD test by SPSS 17.0, with $P<0.05$ indicating significant difference.

\section{Results}

\subsection{The Effects of Different Cryoprotectants Combination on Sperm Motility}

The properties of fresh semen from the three experimental males were evaluated as follows: semen volume $290.0 \pm 14.14$ $(\mathrm{mL})$, sperm concentration $1.78 \pm 0.23\left(\times 10^{9} \mathrm{sperm} / \mathrm{mL}\right)$ and sperm motility $96.3 \pm 1.53(\%)$.

To explore the effects of different cryoprotectants combination on sperm motility at low temperature $\left(4^{\circ} \mathrm{C}\right)$, the changes of sperm motility with storage duration times in the five tested groups were evaluated. The results showed that the effects of all five experimental groups on sperm motility were not significant after $4 \mathrm{~h}$ of in vitro storage. Notably, we found that the sperm motility of undiluted semen (control group) sharply reduced to $4.5 \%$ after $72 \mathrm{~h}$ of in vitro storage, but there was still $30.0 \%$ sperm motility even after $120 \mathrm{~h}$ of in vitro storage in the MT group. Meanwhile, the sperm motility of MT+DMSO groups drastically dropped to $12.3 \%$ after $24 \mathrm{~h}$ of in vitro storage, which might suggest that the DMSO cryoprotectant possibly inhibits sperm motility of A.schrenckii at low temperature $\left(4^{\circ} \mathrm{C}\right)$. Furthermore, $\mathrm{MT}+\mathrm{PG}$ and $\mathrm{MT}+\mathrm{PG}+\mathrm{Y}$ still had higher sperm motility after $72 \mathrm{~h}$ of in vitro storage ( $42.5 \%$ vs. $50.0 \%$, respectively). The effects of the different cryoprotectants combination on sperm motility of A.schrenckii at low temperature $\left(4^{\circ} \mathrm{C}\right)$ are shown in Figure 1. 


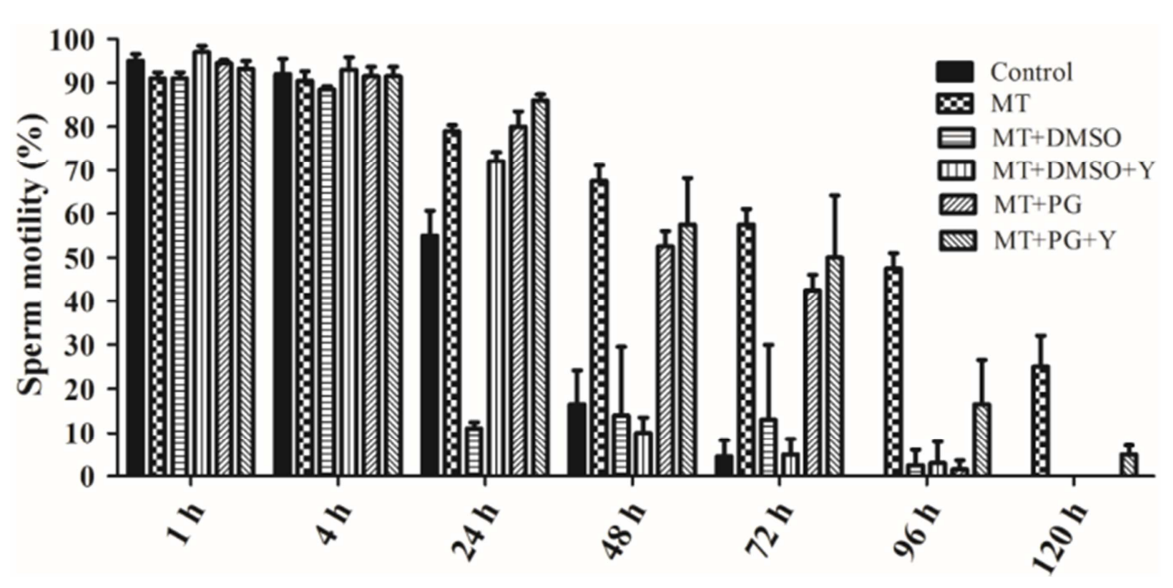

Figure 1. Effects of the different cryoprotectants combination on sperm motility (mean $\pm S D$ ) of A. schrenckii. at low temperature $\left(4^{\circ} \mathrm{C}\right)$.

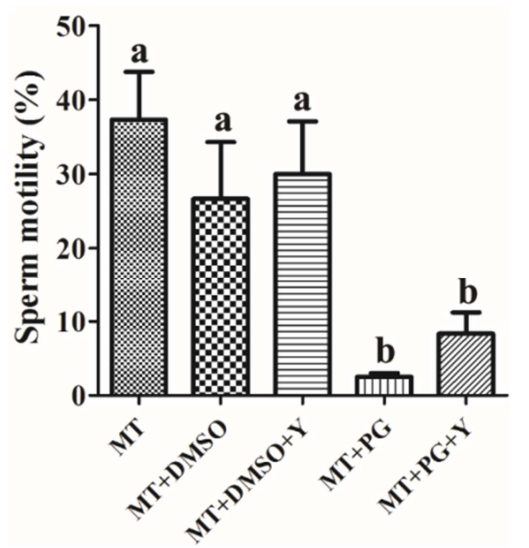

Figure 2. Effect of the different cryoprotectants combination on sperm motility of A. schrenckii. The different letter in each column indicates significant differences $(P<0.05)$.

More importantly, the indexes of the post-thawed sperm motility of five experimental groups were subsequently evaluated. The results indicated that the MT group had the highest post-thawed sperm motility compared to the other four experimental experiments, but the $\mathrm{MT}+\mathrm{PG}$ group had the least sperm motility, only $2.5 \pm 0.5 \%$. Although the two groups with fresh yolk addition $(\mathrm{MT}+\mathrm{DMSO}+\mathrm{Y}$ and $\mathrm{MT}+\mathrm{PG}+\mathrm{Y})$ obtained higher post-thawed sperm motility than the two groups without fresh yolk addition (MT+DMSO and $\mathrm{MT}+\mathrm{PG}$ ), there were no significantly positive effects on sperm motility. The information is in detail summarized in Figure 2.

\subsection{The Effects of Different Cryoprotectants Combination on Fertility Ability and Embryo Development}

About 32-36 h after fertilization, embryo was in yolk plug stage when animal pole was extremely bright yellow and vegetal pole was relatively dark. About $44-46 \mathrm{~h}$, embryo was in the early neural stage, when neural plate began to form in the back of embryo and neural groove appeared. Subsequently, the widest part of neural plate got thicker and neural folds were formed. In the wide neurula plate formation stage, the distinct wide neural plate and $\mathrm{U}$ shaped nerve folds around head neural plate appeared. The development of 32-44 h embryo of A. schrenckii after fertilization is shown in Figure 3(a-c). Meanwhile, the embryos of different experiment groups are also observed in Figure 3(d-f), including MT group, MT+DMSO group and MT+PG group, respectively. The results showed that embryos of $44 \mathrm{~h}$ after fertilization were mainly composed of embryos in above three development stages.
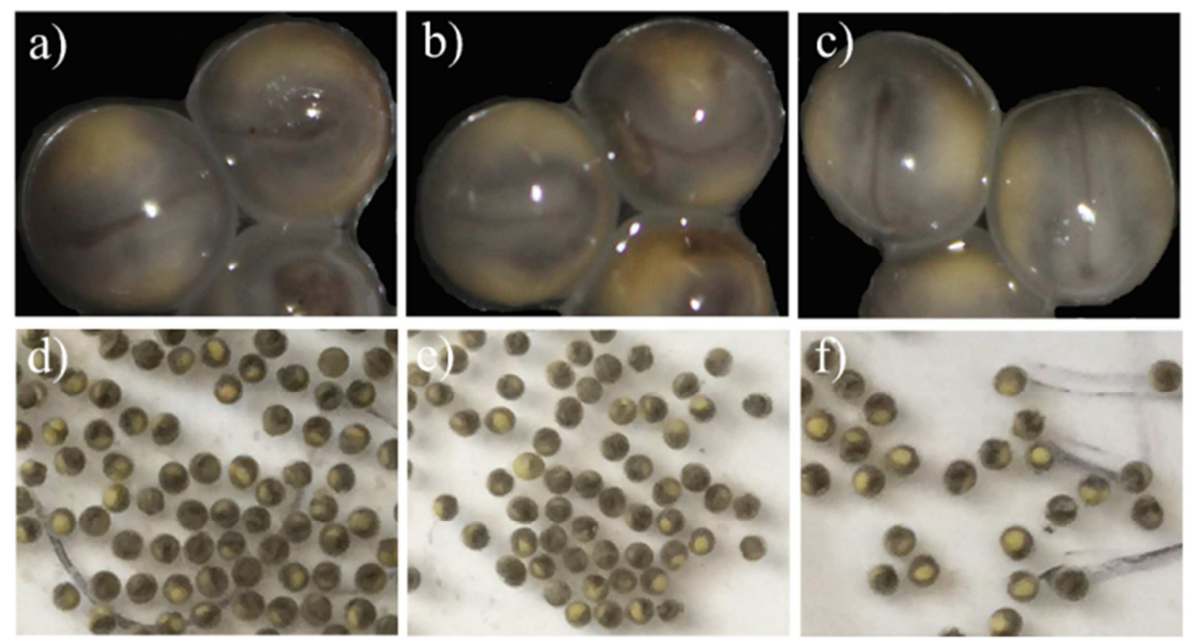

Figure 3. Development observation of 32-44 h embryos of A. schrenckii after fertilization. a) Yolk plug stage. b). Early neurula stage; c) Wide neural plate formation; d) Embryo of MT group; e) Embryo of MT+DMSO group; d) Embryo of MT+PG group. 
The fertilization rates of the post-thawed sperm of the five experimental groups were further evaluated (Figure 4). The results indicated that the fertility rate of the MT group was significantly higher with a value of $42.30 \pm 2.57(\%)$, and it was followed by the MT+DMSO group. Meanwhile, the lowest fertility rate was also observed in the $\mathrm{MT}+\mathrm{PG}$ group.

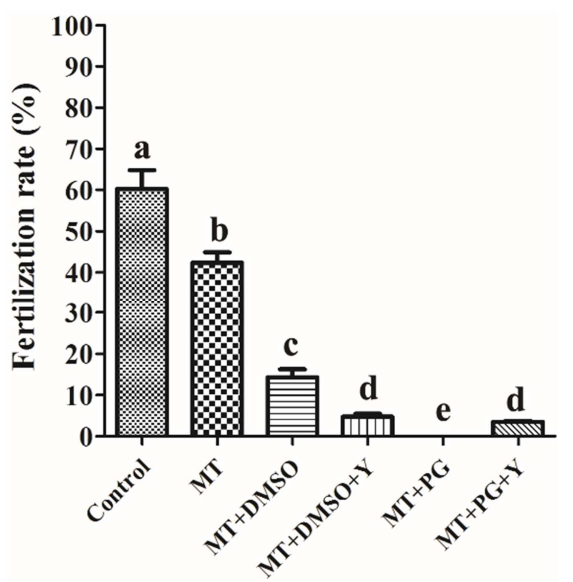

Figure 4. Effect of the different cryoprotectants combination on fertilization rate of A. schrenckii. The different letter in each column indicates significant differences $(P<0.05)$.

The index of hatching rate was compared among five experimental groups (Figure 5). The results indicated that the MT+DMSO group had higher hatching rate than that of the MT group, whereas the hatching rate of the $\mathrm{MT}+\mathrm{PG}$ gorup had the significantly least effect.

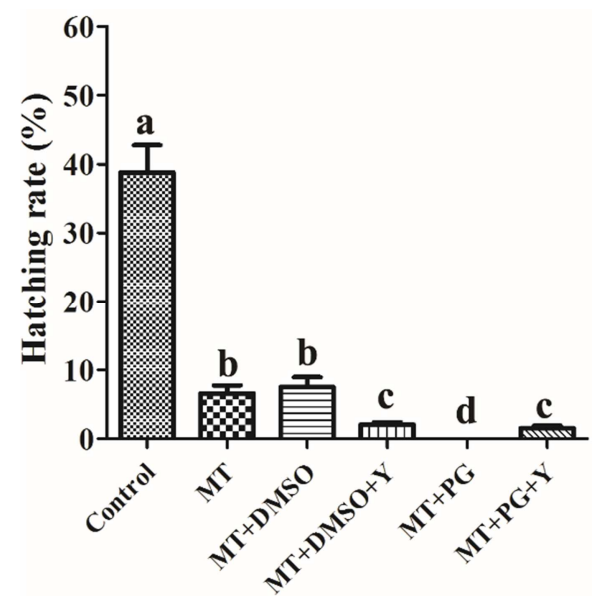

Figure 5. Effect of the different cryoprotectants combination on hatching rate of A. schrenckii. The different letter in each column indicates significant differences $(P<0.05)$.

\section{Discussion}

Sperm cryopreservation is an important technology for preserving genetic information, restoring the population of endangered species and facilitating artificial reproduction in the farming industry. To establish a successful cryopreservation technology, some essential factors need to be assessed, including the cryoprotectant agent, diluent composition, cryoprotectant concentration, cryostraw or cryotube volume, and dilution ratio $[11,12]$. The damages caused by cryopreservation on fish sperm mainly occur during various processes, for example, the concentration of cryoprotectants, freezing temperature and frozen-thaw procedure; based on these factors, the selection and knowledge of the toxicity of cryoprotectant are extremely important. The objective of the present study was to evaluate the effects of cryoprotectants combination on post-thaw sperm motility, fertilization ability and embryogenesis of Amur sturgeon (A.schrenckii).

Sperm motility is a key index for estimating the quality of fresh as well as cryopreserved sperm [12, 13]. Usually, cryopreserved sperm has a lower percentage of motility. For example, after the cryopreservation of turbot sperm, the percentage of motile frozen-thawed sperm was significantly lower than that of fresh sperm [14]. Low motility of frozen-thawed sperm in sturgeon species is often reported (Table 1), such as the higher motile percent $(60 \pm 4.1 \%)$ in Persian sturgeon (A. persicus) [9] and the lower motility rate (only $26 \pm 13 \%$ ) in shortnose sturgeon (A. brevirostrum) [15]. Recently, the Tris-sucrose- $\mathrm{KCl}$ system was regarded as the ideal base medium for sperm cryopreservation in Persian sturgeon [9]. Based on the Tris-sucrose- $\mathrm{KCl}$ system, we modified the cryopreserved medium with supplement of 10 $\mathrm{g} / \mathrm{L} \mathrm{BSA}$ and $5 \mathrm{mM}$ glutamine, which have important roles in membrane protection and the prevention of sperm cell damage during cryopreservation processes. Our results showed that the sperm motility of Amur sturgeon also significantly decreased after cryopreservation, which consolidates that unavoidable cryogenic injuries occur in sturgeon during the freezing and thawing process. Meanwhile, the motility of frozen-thawed sperm in Amur sturgeon was higher than that of stellate sturgeon and shortnose sturgeon, but lower than that of Persian sturgeon, which was probably due to the different freezing methods, such as use of a programmable freezer in sperm cryopreservation of Persian sturgeon.

DMSO is the most widely used cryoprotectant in cryopreservation of fish sperm, and it provides better protection at concentrations between $5 \%$ to $25 \%$ in some marine fish species [16-18]. The embryonic development of $A$. schrenckii can be divided into nine main stages: fertilized egg, cleavage stage, blastula, gastrula, yolk plug, neurula, formation of the optic bud, heart-beat stage, and hatching stages. Especially, the stages form yolk plug to neurula formation indicates successful fertilization and are particularly important stages of embryogenesis. In the present study, after sperm cryopreservation, the MT+DMSO group obtained better effect in terms of the rate of hatched larval fish. The results is interesting and may be explained by a related report, which indicated that DMSO-based vitrificant solutions were suitable for cryopreservation of Persian sturgeon $(A$. persicus) embryos [10]. Moreover, a recent report indicated that the dramatically high level of malondialdehyde (MDA) across the embryonic development may be the main reason leading to a low hatching rate in A. schrenckii [19]. Probably, 
we also speculate that low hatching rate in the MT group may be related with high expression level of MDA. Therefore, MT + DMSO-based method of sperm cryopreservation in Amur sturgeon is worth optimizing. For future studies, it would be desirable to assess the effect using different concentration combination of MT and DMSO as cryoprotectants on sperm cryopreservation of Amur sturgeon.

\section{Conclusions}

The aim of the present study was to examine cryopreservation effects of different cryoprotectants combination on sperm motility, fertilization and embryo development in A. schrenckii. Our results demonstrated that PG as cryoprotectant was certainly unsuitable for sperm cryopreservation of Amur sturgeon. Meanwhile, our results also showed that the post-thawed sperms can support normal embryogenesis for $A$. schrenckii, especial for high fertilization rate at the $32-44 \mathrm{~h}$ after fertilization. More importantly, the MT+DMSO combination obtained optimal hatching edffect, which suggests that MT + DMSO-based method of sperm cryopreservation in Amur sturgeon is worth optimizing.

\section{Abbreviations}

methanol (MT), dimethyl sulfoxide (DMSO), propylene glycol (PG), the International Union for Conservation of Nature Resource (IUCN), luteinizing hormone-releasing hormone analogue (LHRH), fresh yolk (Y).

\section{Declarations of Interest}

The authors declare that they have no competing interests.

\section{Acknowledgements}

We thank Mr. Wenhua Wu for help with Amur sturgeons sampling. This work was supported by the Natural Science Fund of Guangdong Province NO. 2018A030310488, the National Natural Science Fund of China NO. 31802279, the GDAS Special Project of Science and Technology Development NO. 2018GDASCX-0107, and the Planning funds of Scientific and Technological of Guangdong Province NO. 2016B070701016.

\section{References}

[1] Honkala, I. and Lobstein, A., 2008. Identification of Acipenseriformes species in trade. Journal of Applied Ichthyology, 24: 2-19.

[2] Wei, Q.W., Zou, Y., Li, P., and Li, L. 2011. Sturgeon aquaculture in China: progress, strategies and prospects assessed on the basis of nation-wide surveys (2007-2009). Journal of Applied Ichthyology, 27: 162-168.

[3] Li, D., Liu, Z., and Xie, C. 2012. Effect of stocking density on growth and serum concentrations of thyroid hormones and cortisol in Amur sturgeon, Acipenser schrenckii. Fish Physiology \& Biochemistry, 38: 511-520.

[4] Arakawa, T., Carpenter, J. F., Kita, Y. A., and Crowe, J. H. 1990. The basis for toxicity of certain cryoprotectants: A hypothesis. Cryobiology, 27:401-415.

[5] Jun, L. I., Liu, Q., and Zhang, S. 2006. Evaluation of the damage in fish spermatozoa cryopreservation. Chinese Journal of Oceanology and Limnology, 24: 370-377.

[6] Glogowski, J., Kolman, R., Szczepkowski, M., Horváth, Á., Urbányi, B., Sieczyński, P., et al. 2002. Fertilization rate of Siberian sturgeon (Acipenser baeri, Brandt) milt cryopreserved with methanol. Aquaculture, 211: 367-373.

[7] Dzyuba, B., Boryshpolets, S., Shaliutina, A., Rodina, M., Yamaner, G., Gela, D., et al. 2012. Spermatozoa motility, cryoresistance, and fertilizing ability in sterlet Acipenser ruthenus during sequential stripping. Aquaculture, 356-357: 272-278.

[8] Aramli, M. S., Golshahi, K., Nazari, R. M., and Aramli, S. 2015. Effect of freezing rate on motility, adenosine triphosphate content and fertilizability in beluga sturgeon (Huso huso) spermatozoa. Cryobiology, 70: 170-174.

[9] Aramli, M. S., Golshahi, K., Nazari, R. M., Golpour, A., Aramli, S. 2016. Influence of Glutamine Supplementation on Motility and Fertilization Success of Frozen-Thawed Persian Sturgeon (Acipenser persicus) Sperm. Reprod. Domest. Anim., 51: 474-477.

[10] Keivanloo, S., and Sudagar, M. 2016. Cryopreservation of Persian sturgeon (Acipenser persicus) embryos by DMSO-based vitrificant solutions. Theriogenology, 85: 1013-1018.

[11] Suquet, M., Dreanno, C., Fauvel, C., Cosson, J., and Billard, R. 2000. Cryopreservation of sperm in marine fish. Aquaculture Research, 31: 231-243.

[12] Liu, Q., Li, J., Zhang, S., Ding, F., Xu, X., Xiao, Z., et al. 2006. An Efficient Methodology for Cryopreservation of Spermatozoa of Red Seabream, Pagrus major, with 2-mL Cryovials. Journal of the World Aquaculture Society, 37: 289297.

[13] Klimowicz, M. D., Nizanski, W., Batkowski, F., and Savic, M.A. 2008. The comparison of assessment of pigeon semen motility and sperm concentration by conventional methods and the CASA system (HTM IVOS). Theriogenology, 70: 77.

[14] Dreanno C., Suquet M., Quemener L., Cosson J., Fierville F., Normant Y., and Billard. 1997. Cryopreservation of turbot (Scophthalmus maximus) spermatozoa. Theriogenology, 48: 589.

[15] Horvath, A., Wayman, W. R., Urbanyi, B., Ware, K. M., Dean, J.C., and Tiersch, T. R. 2005. The relationship of the cryoprotectants methanol and dimethyl sulfoxide and hyperosmotic extenders on sperm cryopreservation of two North-American sturgeon species. Aquaculture, 247: 243-251.

[16] Fabbrocini, A., Lavadera, S.L., Rispoli, S., Sansone, G. 2002. Cryopreservation of seabream (Sparus aurata) spermatozoa. Cryobiology, 40: 46.

[17] Ding, F., Lall, S. P., Li, J., Lei, J., Rommens, M., and Milley, J.E. 2011. Cryopreservation of sperm from Atlantic halibut (Hippoglossus hippoglossus, L.) for commercial application. Cryobiology, 63: 56-60. 
[18] Liu, Q. H., Ma, D. Y., Xu, S. H., Xiao, Z. Z., Xiao, Y. S., Song, Z.C., et al. 2015. Summer flounder (Paralichthys dentatus) sperm cryopreservation and application in interspecific hybridization with olive flounder ( $P$ olivaceus $)$. Theriogenology, 83: 703-710.
[19] Jin, S., Sun, D., Song, D., Wang, N., Fu, H., Ji, F., et al. 2018. iTRAQ-based quantitative proteomic analysis of embryonic developmental stages in Amur sturgeon, Acipenser schrenckii. Sci. Rep., 8: 6255. 